\title{
PERFORMANCE OF THE CATALYTIC MEMBRANE REACTORS OF DIFFERENT PORE SIZE WITH PALLADIUM AS CATALYTIC PHASE IN HYDROGENATION AND OXIDATION REACTIONS
}

\author{
V. Pinos-Vélez ${ }^{1,2,3}$, F. Medina ${ }^{1 *}$ and A. Dafinov ${ }^{1 *}$ \\ ${ }^{1}$ Chemical Engineering Department, Rovira I Virgili University, Av Paisos Catalans 26, 43007 Tarragona, Spain. \\ ${ }^{2}$ Departamento de Recursos Hídricos y Ciencias Ambientales, Dirección de Investigación (DIUC), Universidad de Cuenca, \\ Av 12 de Abril, Cuenca, Ecuador. \\ ${ }^{3}$ Facultad de Ciencias Química, Universidad de Cuenca, Av 12 de Abril, Cuenca, Ecuador.
}

(Submitted: September 7, 2017 ; Revised: January 6, 2018 ; Accepted: February 19, 2018)

\begin{abstract}
The catalytic membrane reactors (CMR) were prepared using commercial Hollow Fiber Membranes of corundum with $4 \mathrm{~nm}$ or $1400 \mathrm{~nm}$ pores and impregnated with palladium. The tests performed were phenol hydrogenation, hydrogen peroxide generation to oxidize phenol by advanced oxidation processes and chromium (VI) reduction. Reactions were performed in aqueous solution, at mild temperature and under atmospheric pressure. For all tests, the hydrogen passed through the membrane to be activated with palladium. The test with phenol showed that, in the absence of oxygen, the activated hydrogen reacts with the organic matter, leading to the formation of hydrogenated products; in the presence of oxygen, active hydrogen reacts with oxygen to form hydrogen peroxide and subsequently hydroxyl radicals that attack the organic matter until its mineralization. Furthermore, the CMR is capable of reducing chromium (VI) to chromium (III). The CMRs of smaller pore size presented better performance in all reactions.

Keywords: Catalytic membrane reactors; Advanced oxidation processes; Palladium nanoparticles; Hydrogenation; Phenol; Chromium (VI) reduction.
\end{abstract}

\section{INTRODUCTION}

A membrane reactor is a device that combines a membrane separation or distribution process with a chemical reaction in one unit (Lin, 2007). Among the different types of membrane reactors, Catalytic Membrane Reactors (CMRs), devices with catalytic perm-selective membranes or made with an ordinary membrane with a catalyst deposited in or on it, are a good choice to develop efficient and safe catalytic reactions (Caro, 2014; Miachon and Dalmon, 2004). The CMR can be used as a contactor to intensify the contact between reactants and catalysts (Westermann and Melin, 2009). The interfacial contactor mode allows contact between catalyst and the reagents fed from opposite sides of the Hollow Fiber Membrane (Pinos-Vélez et al., 2017). This disposition permits the reaction between reagents in different phases, e.g., liquid and gas with a solid catalyst (Dalmon et al., 2007; Volkov et al., 2011). Catalytic Membrane Reactors in the interfacial contactor mode are therefore good options to develop hydrogenation and oxidation reactions, providing a catalytic interface (Sanchez and Tsotsis, 2002). Using a Hollow Fiber Membrane presents some advantages due to the high chemical, thermal and mechanical resistances of the applied material, in addition to the membrane geometry, which provides a large surface area per

\footnotetext{
*Corresponding autor: Email: anton.dafinov@urv.cat; francesc.medina@urv.cat; veronica.pinos@ucuenca.edu.ec
} 
unit volume of membrane module (Terra et al., 2016).

For instance, Catalytic Membrane Reactors are used in environmental applications to eliminate organic pollutants by advanced oxidation processes (AOP), which are one of the most effective methods because they are a highly competitive water treatment technology for organic pollutants not treatable by conventional techniques (Oller et al., 2011; Poyatos et al., 2010; Pires et al., 2015). These processes are characterized by using the high reactivity of $\mathrm{OH}$. radicals to oxidize the organic matter to obtain less hazardous pollutants or until complete mineralization; AOPs include $\mathrm{O}_{3}, \mathrm{O}_{3} / \mathrm{H}_{2} \mathrm{O}_{2}, \mathrm{UV}, \mathrm{UV} / \mathrm{O}_{3}, \mathrm{UV} / \mathrm{H}_{2} \mathrm{O}_{2}, \mathrm{O}_{3} /$ $\mathrm{UV} / \mathrm{H}_{2} \mathrm{O}_{2}, \mathrm{Fe}^{2+} / \mathrm{H}_{2} \mathrm{O}_{2}$, and photocatalysis (Andreozzi et al., 1999; Mohanty et al., 2017).

One option to develop AOPs using CMRs is to obtain hydrogen peroxide in situ to generate hydroxyl radicals in order to oxidize organic pollutants such as phenol (Osegueda et al., 2012; Osegueda et al., 2015). CMRs are capable of performing the entire Fenton process due to the generation of $\mathrm{H}_{2} \mathrm{O}_{2}$ and formation of $\mathrm{OH}$. radicals for organic matter oxidation. Phenol oxidation comprises different consecutive steps: catechol is the primary oxidation product, indicating that hydroxylation takes place predominantly at the ortho position. The concentration of the other oxidation products, such as hydroquinone (para-hydroxylation), resorcinol (meta-hydroxylation), and p-benzoquinone are less predominant than catechol. The ring opening of the aromatic intermediates leads to the formation of organic acids such as muconic acid, maleic acid, formic acid and malonic acid. As a result, a decrease in the $\mathrm{pH}$ takes place. In fact, all of the intermediates are finally oxidized to formic acid and oxalic acid. Formic acid is also oxidized to $\mathrm{CO}_{2}$ and $\mathrm{H}_{2} \mathrm{O}$, whereas oxalic acid shows quite refractory behavior and may remain in solution (Pignatello et al., 2006).

Palladium is recommended as the catalytic phase to promote the generation of hydrogen peroxide (Remias et al., 2003). In fact, in many studies, Pd has successfully been used to obtain $\mathrm{H}_{2} \mathrm{O}_{2}$ directly from hydrogen and oxygen (Ghedini et al., 2010; Shi et al., 2010; Tomoya et al., 2010).

Another use for palladium catalyst is the hydrogenation of the organic matter such as phenol. It is important to take into account that phenol hydrogenation typically involves high temperature and pressure (Matos and Corma, 2011; Pérez et al., 2011; Watanabe and Arunajatesan, 2010). Commonly, in such reactions, organic solvents are used: such as ethyl alcohol, cyclohexane, ionic liquids, etc. (Liu et al., 2009; Liu et al., 2007; Maksimov et al., 2013). Most of the mechanisms are tested in the vapor or gas phase (Claus et al., 2000; Mahata et al., 2001; Park and Keane, 2003; Sikhwivhilu et al., 2007). Nevertheless, some work has been reported on the hydrogenation of phenol in mild conditions (Cheng et al., 2014; Morales et al., 2002; Wang et al., 2011; Zhu et al., 2014).

Our previous works have demonstrated the capability of CMRs with palladium to develop advanced oxidation processes and hydrogenation with a single device, changing the reaction conditions and reagents (Pinos-Vélez et al., 2016b). Indeed, this article is a continuation of such previous work to achieve a deeper understanding of the different reactions achieved using the same device and the influence of the pore size of the CMRs.

Another application of the Catalytic Membrane Reactors with palladium is the reduction of toxic chromium (VI) in water to chromium (III), with less toxicity, to levels below 50 ppb (Pinos-Vélez et al., 2016a). Hence this paper points out the difference in performance when CMRs with different pore sizes are used.

Thus, the objective of this work was to evaluate the capability of Catalytic Membrane Reactors of different pore size with palladium as a catalytic phase, in treatments of wastewater polluted with organic matter or inorganic compounds as well as in hydrogenation reactions using phenol or chromium (VI) in aqueous solution as model pollutant or reagent, in very mild conditions.

\section{EXPERIMENTAL}

\section{Catalytic Membrane Reactor preparation}

Corundum Hollow Fiber Membranes (HFM) made of alpha alumina ( $\alpha-\mathrm{Al} 2 \mathrm{O} 3)$ for ultra and nano filtration manufactured by Ceparation ${ }^{\mathrm{TM}}$ of $4 \mathrm{~nm}$ and $1400 \mathrm{~nm}$ porous size were used as the starting material. The porosity of the membranes varied from $10 \%$ to $15 \%$. The fibers have an inner diameter of $2 \mathrm{~mm}$ and an outer diameter of $3 \mathrm{~mm}$ with a total length of $300 \mathrm{~mm}$. Each of the Catalytic Membrane Reactors was prepared by using a $150 \mathrm{~mm}$ fragment of the membrane. The procedure to prepare Catalytic Membrane Reactors of corundum impregnated with palladium has been described in previous works (Pinos-Vélez et al., 2016a; Pinos-Vélez et al., 2016b). Briefly, the precursor solution of palladium salt, $\mathrm{PdCl}_{2}$ (Johnson Matthey) with $59.83 \%$ of the noble metal, was added into Milli-Q water, while stirred. Then, concentrated hydrochloric acid (37\%, Sigma-Aldrich) was added in a drop-wise manner into the solution until the salt was completely dissolved. Finally, the concentration was adjusted with Milli-Q water in order to achieve approximately $1 \%$ of weight of $\mathrm{Pd}$ per weight of the membrane. The palladium precursor solution was used to impregnate the membranes of 1400 or $4 \mathrm{~nm}$. 
Once the impregnation was complete, the Catalytic Membrane Reactors were dried in a special vessel in a vacuum for $30 \mathrm{~min}$. In this step, the CMR was rotated along the horizontal axis in order to avoid preferential deposition of the salts onto the membrane. The CMR was dried at $120^{\circ} \mathrm{C}$ for $5 \mathrm{~h}$ and finally calcined at $450^{\circ} \mathrm{C}$ overnight. The palladium loaded onto the CMR was further activated by reduction under $20 \mathrm{sccm} / \mathrm{min}$ flowing hydrogen at $350^{\circ} \mathrm{C}$ for 3 hours. The amount of palladium deposited into the Hollow Fiber Membranes was calculated by the weight difference between the original and the modified membrane. The data for the CMRs obtained are summarized in Table 1.

Table 1. Composition of the Catalytic Membrane Reactors.

\begin{tabular}{lccc}
\hline \multicolumn{1}{c}{ CMR } & Pore size $(\mathbf{n m})$ & \% Pd & \% Porosity \\
\hline CMR1400 & 1400 & 0.9 & 15 \\
CMR4 & 4 & 0.75 & 10 \\
HFM1400 & 1400 & - & 15 \\
HFM4 & 4 & - & 10 \\
\hline
\end{tabular}

CMR, Catalytic Membrane Reactor; HFM, Hollow Fiber Membranes.

\section{Characterization of catalytic membrane reactors}

Transmission electron microscopy (TEM) Transmission electron microscopy was employed to examine the $\mathrm{Pd}$ nanoparticles in the catalytic membrane reactor. The equipment used was a transmission electron microscope, TEM, JEOL model 1011 from Servei de Recursos Científics i Tècnics of URV. A piece of the CMR was crushed and the powder was dispersed in ethanol for the samples obtained by impregnation, sputtering and microemulsion. The nanoparticles obtained by the polyol route without the support were analyzed directly. Then, a drop of the sample in suspension was placed onto a copper grid. The grid was dried and placed in the TEM to be observed. The sizes of the observed Pd particles were determined using the ITEM software by Olympus.

X-ray diffraction (XRD) - To identify the palladium and corundum crystals, pieces of the catalytic membrane reactor were analyzed using X-ray diffraction. XRD measurements were performed using a Bruker-AXS D8-Discover diffractometer equipped with a parallel incident beam (Göbel mirror), a vertical $\theta-\theta$ goniometer, an $\mathrm{XYZ}$ motorized stage and a General Area Diffraction System (GADDS). Samples were placed directly on the sample holder and the area of interest was selected with the aid of a video-laser focusing system. An X-ray collimator system allowed the analysis of $500 \mu \mathrm{m}$ areas. The $\mathrm{X}$-ray diffractometer was operated at $40 \mathrm{kV}$ and 40 $\mathrm{mA}$ to generate $\mathrm{CuK}$ radiation. The GADDS detector was a HI-STAR (multiwire proportional counter of 30 x $30 \mathrm{~cm}$ with 1024 x 1024 pixels) placed $15 \mathrm{~cm}$ away from the sample. A frame (2D XRD patterns) covering
$24-562 \theta$ was collected. The exposure time was 900 s per frame and it was chi-integrated to generate the conventional $2 \theta$ vs. intensity diffractogram. Image scale: small lines separation corresponds to $\approx 100$ $\mu \mathrm{m}$. The average area analyzed was represented by an ellipsoid centered in the cross with a constant short axis of $0.5 \mathrm{~mm}(\mathrm{~N}-\mathrm{S}$ direction) and a variable long axis (from 1.5 to $0.6 \mathrm{~mm}$ in the W-E direction). The analysis of the XRD diffractogram was performed by the ICDD database (release 2007) using Diffracplus Evaluation software (Bruker 2007).

\section{Tests}

Test of generation of hydrogen peroxide - Both CMR were introduced in a vessel containing $100 \mathrm{~mL}$ Milli-Q water. Hydrogen flow of $6 \mathrm{sccm} / \mathrm{min}$ was supplied to one end of the CMR whilst the other end was kept closed. In this way, the hydrogen passed across the membrane wall reaching the external surface. Air was bubbled into the water as an oxygen source in the reactor vessel. High performance liquid chromatography, a Shimadzu HPLC equipped with LC $20 \mathrm{AB}$ system and with a diode array detector SPDM10Avp, was used. Hydrogen peroxide was analyzed at a wavelength of $193 \mathrm{~nm}$ after direct injection of the sample without a column. The mobile phase was Milli-Q water, $1 \mathrm{~mL} / \mathrm{min}$.

Test of treatment of $100 \mathrm{ppm}$ of phenol in aqueous solution - A $100 \mathrm{ppm}$ synthetic water solution of phenol was prepared using Milli-Q water and phenol (98.5\%, Riser S.A). The Catalytic Membrane Reactors were tested with $100 \mathrm{ppm}$ of phenol in water solution in semi-batch mode. The hydrogen flow was adjusted using a mass flow controller and supplied to one end of the reactor while the other end was kept closed. The hydrogen supply was $6 \mathrm{sccm} / \mathrm{min}$. The hydrogen crossed the membrane wall reaching the external surface. The CMR was submerged into a glass reactor containing $100 \mathrm{~mL}$ of the phenol-water solution. The tests were performed at $60^{\circ} \mathrm{C}$ for 7 hours at atmospheric pressure. Thereafter, the experiments were divided into three groups: (a) hydrogenation with no external gas supply, and with Ar supply as sweep gas; (b) oxidation with pure oxygen supply; (c) oxidation with air supply to the reaction vessel containing the model solution. In order to saturate the solution when an external gas was supplied, this gas was bubbled into the vessel 10 minutes before introducing the membrane with hydrogen. The experimental set ups are shown in Figure 1.

In order to determine the elimination of the organic substances by stripping, individual tests for phenol, cyclohexanol and cyclohexanone were done. The stripping test for phenol was done in the same condition as the oxidation tests with air and with Argon, but using 


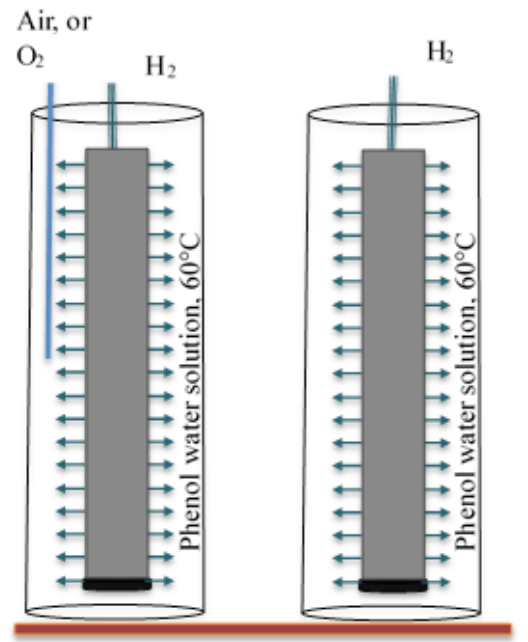

Figure 1. Set up for the oxidation and hydrogenation reaction using a water solution of phenol as model compound.

a Hollow Fiber Membranes without palladium. The stripping tests for cyclohexanol and cyclohexanone were done with a Hollow Fiber Membrane in the same conditions of the hydrogenation tests but using argon as a sweep gas.

High performance liquid chromatography, Shimadzu HPLC equipped with LC 20AB system and with a diode array detector SPD-M10Avp, was used to measure the concentrations of phenol as well as the intermediates in phenol oxidation. A C-18 column (ID. $4.6 \mathrm{~mm}$, length $250 \mathrm{~mm}$, Omnisphere, Varian) was used to separate the reaction intermediate products from the phenol. The concentrations of phenol during the tests were measured every hour. The analysis of the phenol was made at a wavelength $254 \mathrm{~nm}$. The mobile phase of $1 \mathrm{~mL} / \mathrm{min}$ was a mixture of $60 \%$ water at $\mathrm{pH}$ 3 adjusted with acetic acid and $40 \%$ of acetonitrile (Aldrich).

The total organic carbon was measured with a Shimadzu TOC-L CSN equipment. Only the initial and the final samples were assessed.

The hydrogenated products at the end of each experiment were extracted from the aqueous solutions, previously saturated with $\mathrm{NaCl}$, with ethyl acetate (Sigma). Hydrogenation products of phenol extracted were assessed using the gas chromatograph Shimatzu GC-2010 with ZEBRON ZB-WAX column and FID detector. The analyses GC conditions were: $115 \mathrm{kPa}$ head column pressure, helium flow $144 \mathrm{~mL} / \mathrm{min}$, split ratio 15 , injector and detector temperature $250^{\circ} \mathrm{C}$. The temperature ramp was from $60^{\circ} \mathrm{C}$ to $180^{\circ} \mathrm{C}$ and held for $5 \mathrm{~min}$. The obtained values were corrected by a relative response factor (Rome et al., 2012). A calibration curve was prepared for phenol, cyclohexanone (Fluka), for cyclohexanol (Sigma-Aldrich) and for 2-cyclohexen1-one (Aldrich), from 0.5 to $100 \mathrm{ppm}$ and $\mathrm{R}$ of 0.999 .
In the reaction vessel, the dissolved oxygen was measured in each experiment using a SG6 (MettlerToledo) dissolved oxygen meter.

Chromium Reduction - In order to test the dissimilarities between CMRs of different pore size, experiments to achieve the reduction $\mathrm{Cr}$ (VI) to $\mathrm{Cr}$ (III) were performed. The procedure was previously described elsewhere (Pinos-Vélez et al., 2016a). A water solution of $8 \mathrm{mg} / \mathrm{L}$ of $\mathrm{Cr}$ (VI) was prepared from $\mathrm{K}_{2} \mathrm{CrO}_{4}$ salt (Panreac) and adjusted to $\mathrm{pH}$ 3. Samples were prepared in accordance with the standard methods $3500-\mathrm{Cr}$ (colorimetric method for the examination of water and wastewater) (Apha, 2012). 1,5-biphenyl carbazide was added to the samples as an indicator and its absorbance was measured at $540 \mathrm{~nm}$ each hour using the Jasco V-630 Spectrophotometer.

\section{RESULTS AND DISSCUSION}

The Catalytic Membrane Reactors obtained are described in Table 1. The Catalytic Membrane Reactors achieved have a palladium concentration around $1 \%$ considering the weight of the Hollow Fiber Membranes. The porosity of the CMRs did not differ from the porosity of the original Hollow Fiber Membrane.

\section{Characterization}

In Figure 2, spherical dark brown palladium nanoparticles can be seen over corundum. The results obtained through the TEM showed a mean nanoparticle size of $6 \mathrm{~nm}$ with a standard deviation of $2 \mathrm{~nm}$. No differences were found between palladium nanoparticles loading in CMR4 and CMR1400.

Figure 3 shows the XRD analysis; in HFM (a), only the characteristics peaks of corundum were found

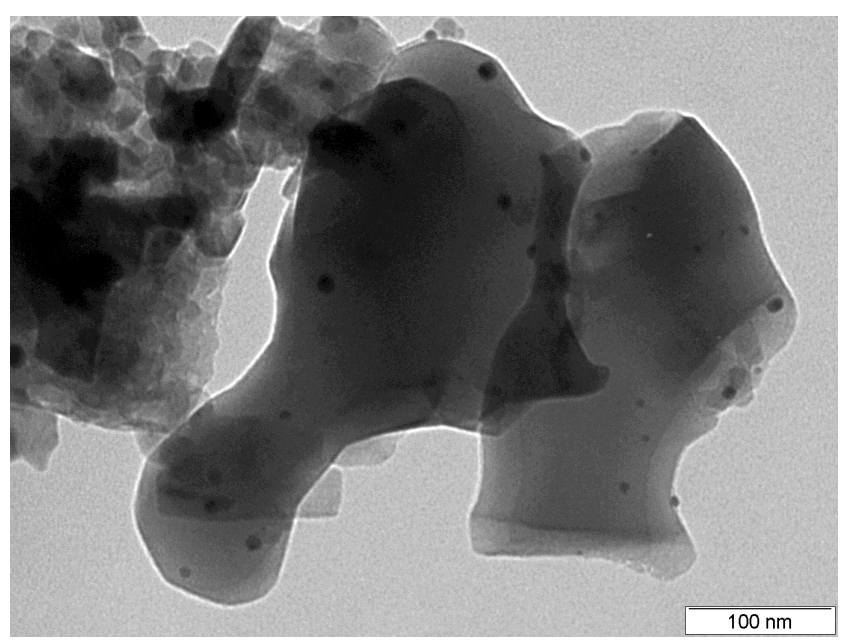

Figure 2. Palladium nanoparticles by impregnation of Hollow Fiber Membrane of Corundum. 


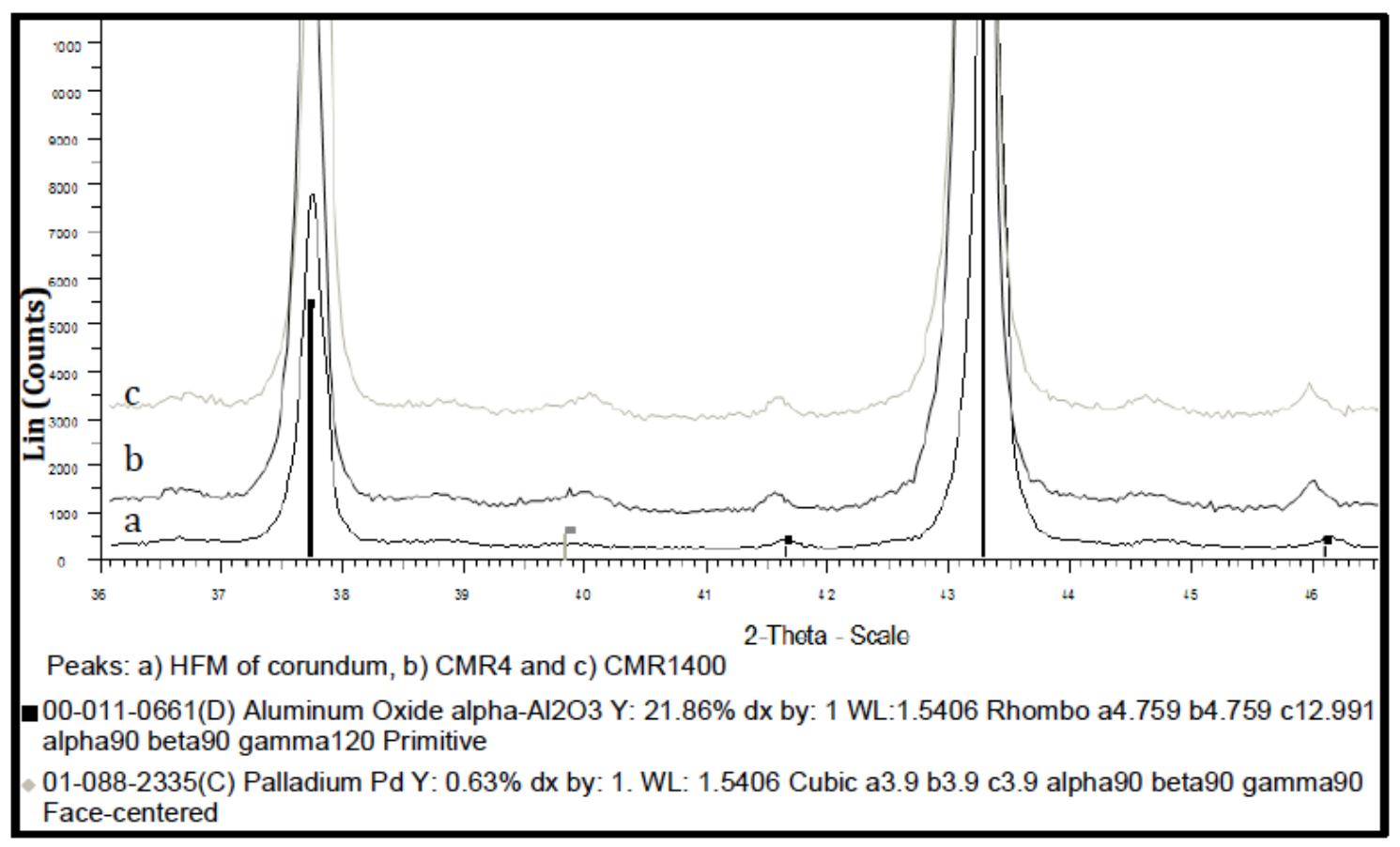

Figure 3. XRD analysis of CMR4 and CMR1400.

as was expected. In the CMR samples (b and c), the characteristic peaks of corundum and palladium can be seen at 39.9 degrees for palladium and at 37.7, 41.65, 43.3 and 46.1 degrees for corundum. No differences between membranes can be found.

\section{Hydrogen Peroxide generation}

TheCMRstestedreached themaximum concentration of $\mathrm{H}_{2} \mathrm{O}_{2}$ after twenty minutes. Subsequently, no more hydrogen peroxide was produced. A possible reason to explain this apparent inactivity can be attributed to palladium deactivation caused by the $\mathrm{H}_{2} \mathrm{O}_{2}$ produced (Osegueda et al,. 2012). The results of this test are presented in Table 2 . The reaction pathway proposed is the hydrogen passing through the CMR where a dissociative absorption is produced. The atomic hydrogen reacts with the oxygen dissolved in the water to form hydrogen peroxide and water.

CMR1400 gave the best performance and, as was expected, the Hollow Fiber Membranes did not react. In both cases, CMR 1400 and CMR 4 produced enough $\mathrm{H}_{2} \mathrm{O}_{2}$ to be tested in Advanced Oxidation Processes to eliminate phenol.
A possible drawback in the process is the peroxide decomposition caused by the palladium. In order to check this, once a maximum in the peroxide concentration was reached, the hydrogen supply was stopped and the $\mathrm{H}_{2} \mathrm{O}_{2}$ concentration was monitored. No variation in the peroxide concentration was measured for at least $20 \mathrm{~min}$.

In order to activate the CMRs, the reactors were removed from the reaction vessel and cleaned with Milli-Q water. Then, hydrogen was passed through the membrane for 10 minutes.

\section{Phenol test}

The results of the tests can be seen in Table 3 and Figure 4. All tests were performed at $60^{\circ} \mathrm{C}$ and atmospheric pressure in semi-batch mode at neutral $\mathrm{pH}$. The only exception was the test where $\mathrm{Fe}_{2}\left(\mathrm{SO}_{4}\right)_{3}$ was added to improve the oxidation performance; in this case, the $\mathrm{pH}$ was set to 2 with hydrochloric acid to maintain the iron solubilized. HFM 1400 and HFM 4 were tested as blanks using $\mathrm{H}_{2}$ that passed through the membrane and bubbling air. In the blank tests, leaching of palladium was measured by ICP MS; no

Table 2. Hydrogen peroxide generation at room conditions with the CMRs.

\begin{tabular}{|c|c|c|c|c|c|}
\hline CMR & $\begin{array}{c}\text { Trans-membrane } \\
\text { pressure } \\
\text { (barg) }\end{array}$ & $\underset{\mathrm{Mg} / \mathrm{L}}{\operatorname{Max} \mathrm{H}_{2} \mathrm{O}_{2}}$ & $\begin{array}{c}\% \text { Efficiency } \\
\mathrm{mol} \mathrm{H}_{2} \mathrm{O}_{2} / \mathrm{mol} \mathrm{H}_{2}\end{array}$ & $\begin{array}{l}\operatorname{mol~H}_{2} \mathrm{O}_{2} / \\
\text { h.mol Pd* }\end{array}$ & $\begin{array}{c}\operatorname{mol~H}_{2} \mathrm{O}_{2} / \\
\text { h.m membrane }\end{array}$ \\
\hline CMR1400 & 2.3 & 11 & 0.45 & 0.57 & 0.06 \\
\hline CMR4 & 3.7 & 7 & 0.22 & 0.34 & 0.03 \\
\hline HFM1400 & 0.6 & 0 & 0 & 0 & 0 \\
\hline HFM4 & 3.4 & 0 & 0 & 0 & 0 \\
\hline
\end{tabular}

* Initial hydrogen peroxide generation rates measured in the first ten minutes of the tests. 
Table 3. Results of the test of the phenol oxidation and hydrogenation.

\begin{tabular}{|c|c|c|c|c|c|c|}
\hline \multirow{2}{*}{ CMR } & \multirow{2}{*}{ Test } & \multirow{2}{*}{$\begin{array}{c}\mathrm{mg} / \mathrm{L} \\
\mathrm{DO}\end{array}$} & \multirow{2}{*}{$\begin{array}{c}\mathrm{mg} / \mathrm{L} \\
\text { Conversion, } \\
\text { LC } \\
\end{array}$} & \multicolumn{3}{|c|}{$\%$ Selectivity } \\
\hline & & & & Mineralization, TOC & $\mathbf{C}=\mathbf{O}, \mathbf{G C}$ & C-OH, GC \\
\hline 1400 & $\mathrm{H}_{2}$ & \multirow{5}{*}{0.34} & 26 & 00 & - & - \\
\hline $4^{*}$ & $\mathrm{H}_{2}$ & & 44 & 00 & 52 & 22 \\
\hline 4 & $\mathrm{H}_{2}, 25^{\circ} \mathrm{C}$ & & 16 & 00 & - & - \\
\hline 1400 & $\mathrm{H}_{2}, \mathrm{Ar}$ & & 25 & 56 & - & - \\
\hline 4 & $\mathrm{H}_{2}, \mathrm{Ar}$ & & 52 & 73 & 19 & 07 \\
\hline 1400 & $\mathrm{O}_{2}, \mathrm{H}_{2}$ & \multirow{2}{*}{23.6} & 32 & 59 & 00 & 00 \\
\hline $4 *$ & $\mathrm{O}_{2}, \mathrm{H}_{2}$ & & 39 & 68 & 00 & 00 \\
\hline 1400 & $\mathrm{H}_{2}$, Air bubble & \multirow{6}{*}{5.56} & 34 & 43 & 12 & 06 \\
\hline $4 *$ & $\mathrm{H}_{2}$, Air bubble & & 53 & 67 & 09 & 05 \\
\hline 1400 & $\mathrm{H}_{2}$, Air by diffusor & & 36 & 46 & - & - \\
\hline 4 & $\mathrm{H}_{2}$, Air by diffusor & & 52 & 26 & 38 & 11 \\
\hline 1400 & $\mathrm{H}_{2}$, Air, $\mathrm{Fe}$ & & 42 & 58 & - & - \\
\hline 4 & $\mathrm{H}_{2}, \mathrm{Air}, \mathrm{Fe}$ & & 61 & 71 & 23 & 00 \\
\hline
\end{tabular}

Dissolved oxygen, DO; cyclohexanone, $\mathrm{C}=\mathrm{O}$, cyclohexanol C-O; * values taken from Pinos-Vélez et al. (2016b).
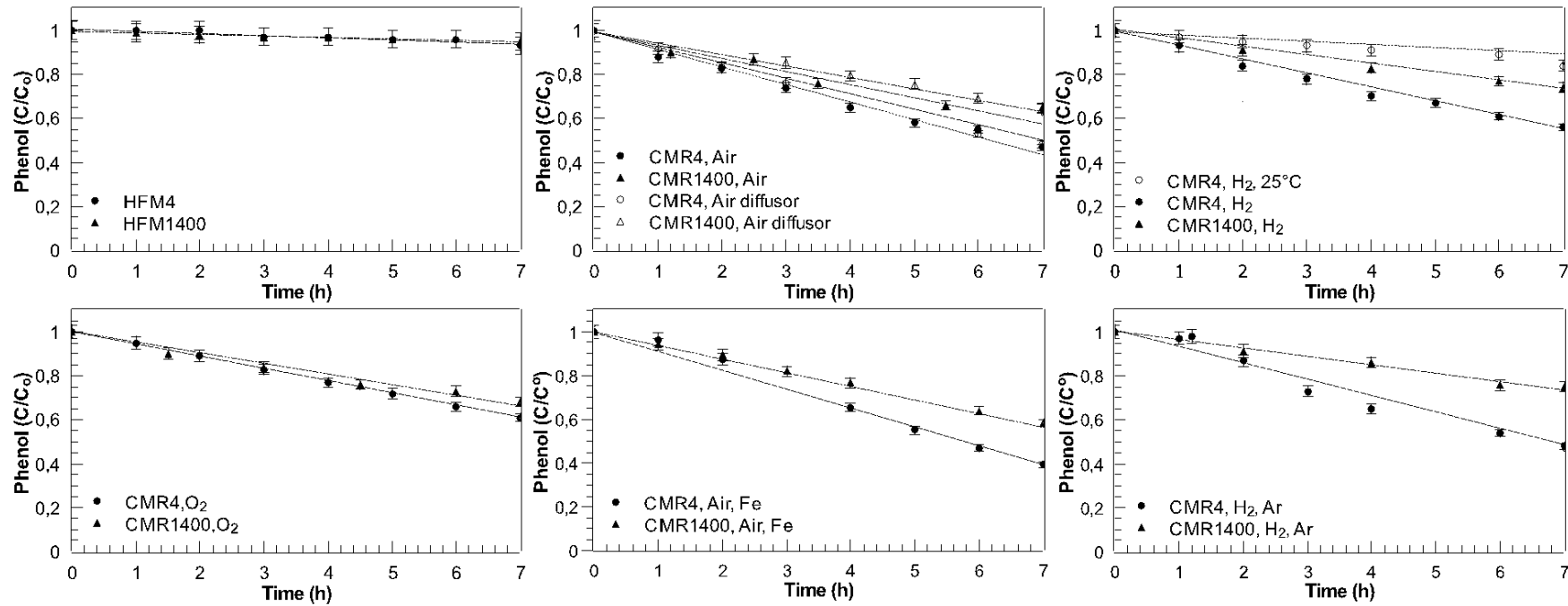

Figure 4. Tests of hydrogenation and Advanced oxidation processes using $100 \mathrm{ppm}$ of phenol in aqueous solution at $60^{\circ} \mathrm{C}$ and atmospheric pressure.

leaching of palladium was detected. The reaction rate fit best with a zero order reaction with respect to the phenol concentration.

In linear regressions for the hydrogenation reaction, the $\mathrm{R}^{2}$ obtained was 0.987 for CMR4 and 0.992 for CMR1400; when argon was added into the reaction, the $\mathrm{R}^{2}$ was 0.966 for CMR4 and 0.984 for CMR1400; for the test of hydrogenation at atmospheric temperature $\mathrm{R}^{2}$ was 0.968 . In the reaction with hydrogen and oxygen, the $\mathrm{R}^{2}$ was 0.999 for CMR4 and 0.980 for CMR1400; in the reaction with hydrogen and air, the $\mathrm{R}^{2}$ was 0.984 for CMR4 and 0.951 for CMR1400, when the diffusor was used, the values were 0.997 for CMR4 and 0.998for CMR1400. Finally, in the test where hydrogen, air, and $\mathrm{Fe}$ were added, the values were 0.992 for CMR4 and 0.998 for CMR1400.

The CMRs prepared with a ceramic Hollow Fiber Membrane and Pd as the active phase are able to catalyze different reactions in one set-up, depending on the operational conditions and reagents. This proves that the CMRs are capable of developing different reactions due to their versatility (Sanchez and Tsotsis, 2002).

Table 3 and Figure 4 show that both CMR successfully reacted in each condition. Despite the lower content of $\mathrm{Pd}$, in the hydrogenation reaction, CMR4 obtained better results. Hence, a smaller pore size favors the reaction due to improved hydrogen diffusion. Cyclohexanone and cyclohexanol were found as hydrogenation products. In the test at $25^{\circ} \mathrm{C}$, very poor results were obtained, almost comparable with the blank results obtained with the HFMs. The hydrogenation tests with phenol were performed again, but using argon as sweep gas. Surprisingly, the total organic carbon decreased in the same sense of the amount of phenol; furthermore, less hydrogenation products were found in comparison of the hydrogenation tests without Ar, see Table 3. Then, stripping tests with phenol, cyclohexanol and cyclohexanone were performed, see Figure 5. The tests 


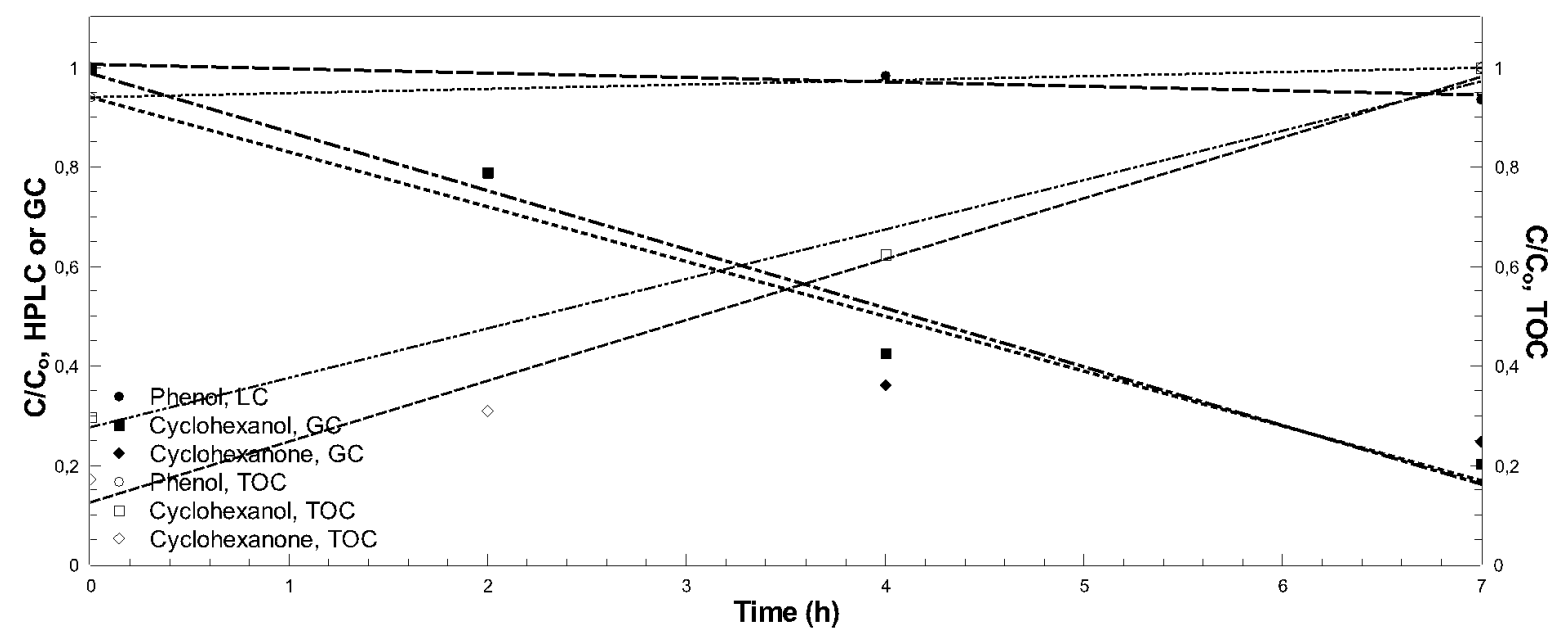

Figure 5. Stripping tests of phenol, cyclohexane and cyclohexanol in aqueous solution at $60^{\circ} \mathrm{C}$ and atmospheric pressure. Hydrogen was passed through HFMs, and argon was bubbled as sweep gas.

found that the cyclohexanone and cyclohexanol were easily stripped. In order to determine the stripping in each reaction, a connection to collect the gases of the reaction, with a known amount of ethyl acetate, was adapted in the set up. The ethyl acetate was analyzed by gas chromatography. The reactions using the CMRs were repeated with this new set up; cyclohexanone and cyclohexanol were found in the sample corresponding of the stripping. A little conversion variation between the test with no external gas and with Ar was found; that is, when a product is eliminated, the equilibrium will be recovered and generates more of the eliminated product.

Fenton reactions to oxidize phenol were performed using a) oxygen, b) air as oxygen source, and c) air and $\mathrm{Fe}$ to improve the reaction. In the reaction with bubbled oxygen, no important difference was found between the performances of the CMRs. Between them, CMR4 achieved a better conversion. In the samples to corroborate stripping, no hydrogenation products were found; in the extracted final samples, hydrogenation products were under the detection limit. Furthermore, through HLPC, typical intermediates of phenol oxidation were found, such as catechol, hydroquinone, resorcinol, and p-benzoquinone (Pignatello et al., 2006). Hence, we can conclude that only oxidation is produced when oxygen is used due to the large amount of this gas available in the reaction.

In the oxidation test using air as oxygen source, the CMR4 achieved the best performance in the three variations of the test, which are air supply i) by tube or ii) by diffuser, or iii) air by tube and added iron to improve the Fenton reaction. There was no difference due to the mode of the air supply. This result is explained by the fact that oxygen used in the reaction is the dissolved oxygen; thus, the amount of oxygen depends on the temperature and pressure. In fact, when the amount of oxygen in the water was measured, no differences were found by way of supply. In all varieties of the tests, apart from intermediates of phenol oxidation, hydrogenation products were found. Hence, hydrogenation and oxidation compete in these conditions. Moreover, in the samples to detect stripping, hydrogenation products were found. This mean that not all the Total Organic Carbon eliminated in these reactions is due to phenol mineralization; part of this is due to phenol hydrogenation products being stripped. Regarding the amount of hydrogenation products, a lower quantity was found when the air was bubbled; apparently, the small bubbles originated by the diffusor are less efficient to produce stripping. Since gas transfer occurs through the gas-liquid interface, this operation has to be carried out so as to maximize the opportunity of interfacial contact between the two phases (Glória et al., 2016). In the reaction when iron is added, more conversion was found because iron enhances the hydroxyl radical generated from hydrogen peroxide and promoted the phenol oxidation. Once again, hydrogenation and oxidation products were found. In conclusion, when air is supplied as the oxygen source hydrogenation and oxidation compete. Therefore, it is recommended to use oxygen and not air if an advanced oxidation process is required. This result is reasonable because oxygen is more soluble in water than air; in fact, at $60^{\circ} \mathrm{C}$ the Henry constant for oxygen is 62200 Pascal, while for air it is 100500 Pascal; furthermore, one must take into account that air content is approximately $21 \%$ oxygen. A disadvantage to use oxygen instead of air is that the treatment cost increases; hence, an optimization of the process is needed.

The reaction pathway proposed is hydrogen activation by the palladium nanoparticles over the HFM. This atomic hydrogen can react with the oxygen in water to generate hydrogen peroxide. As a result, $\mathrm{H}_{2} \mathrm{O}_{2}$ releases hydroxyl radical due to $\mathrm{Pd}$ or Fe. 


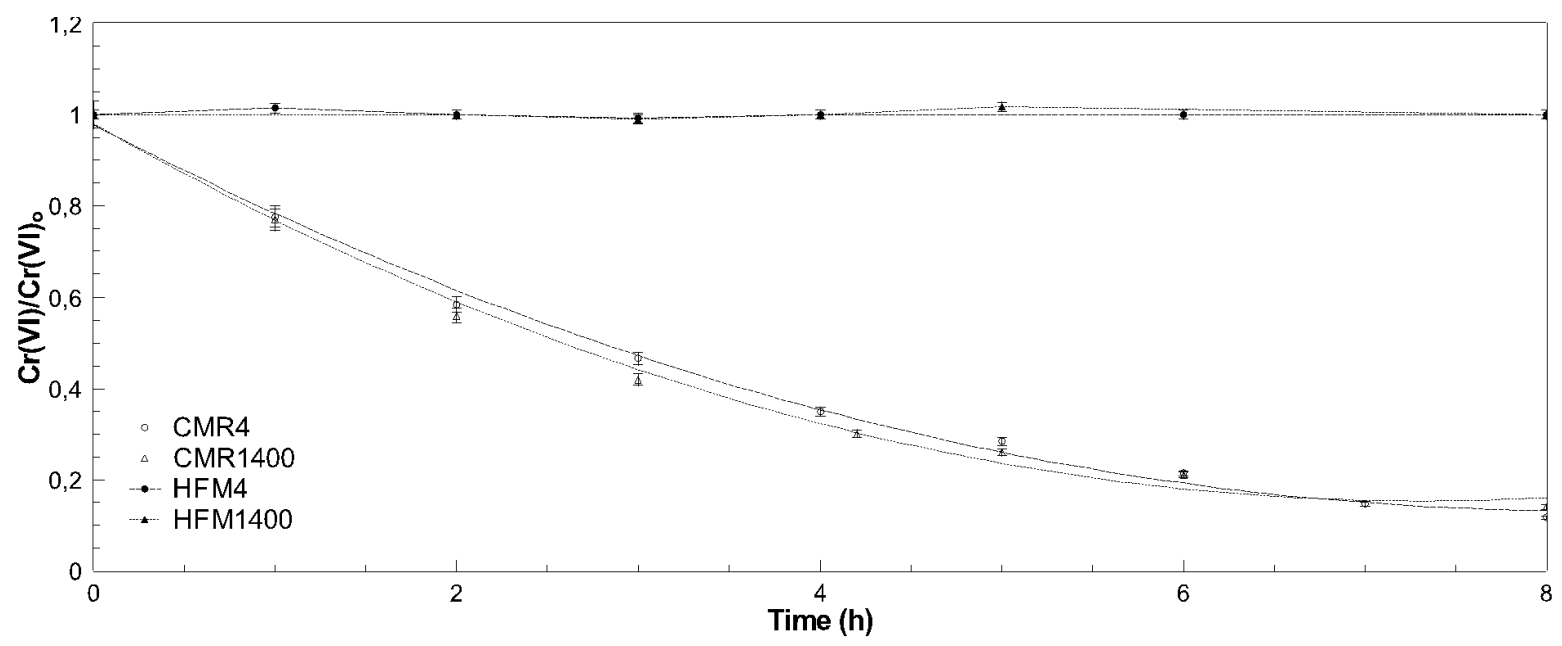

Figure 6. Chromium (VI) reduction to Chromium (III) at atmospheric conditions, $\mathrm{pH}=2$.

These radicals attack the organic matter. In this case, phenol degrades through the formation of catechol, hydroquinone, resorcinol, and p-benzoquinone until mineralization occurs. Alternatively, the activated hydrogen can react directly with the organic matter to produce the hydrogenation products, cyclohexanone and cyclohexanol. Therefore, when hydrogenation is required, only hydrogen should be used in the test, but when an advanced oxidation process is needed, oxygen must be used.

\section{Chromium reduction}

The results of the test of chromium (VI) reduction to chromium (III) are presented in Figure 6. No differences in performance were found between CMR1400 and CMR4. The calculated rate of $\mathrm{Cr}(\mathrm{VI})$ reduction for the first $4 \mathrm{~h}$ was $1.4 \mathrm{ppm} / \mathrm{h}$ in both reactors in similar experimental conditions. In the case of CMR 4 , the amount of Pd, $0.75 \%$, is lower than that of CMR1400, $0.9 \%$. Considering this difference, it seems that the CMR with a lower nominal pore size is more active. However, considering the differences between the pore sizes of both reactors, the obtained activity is lower than expected. This result can be attributed to the lack in uniformity of the pore size distribution of the starting membrane. Another possibility is that the chromate reduction in this concentration and experimental conditions is kinetically limited.

The reaction results best fit with a first-order reaction with respect to the chromium concentration, as can be seen in Figure 4. $\mathrm{R}^{2}$ obtained for these cases were 0.996 for CMR4 and 0.995 for CMR1400. From the results obtained with the CMRs, it can be deduced that part of the hydrogen gas flowing through the porous membrane matrix is activated on the $\mathrm{Pd}$ surface. Thereafter, the activated hydrogen reacts with the chromate anions dissolved in the water, reducing them. The reaction takes place predominantly at the solid-liquid-gas contact point (Pinos-Vélez et al., 2016a).

\section{CONCLUSIONS}

All prepared CMRs appeared to be active for generation of hydrogen peroxide directly from hydrogen and oxygen when tested in contactor interfacial mode at ambient conditions. Moreover, the catalytic membrane reactors with a single $\mathrm{Pd}$ catalytic phase are active either for phenol oxidation or for its hydrogenation. The reactions can be carried out under mild conditions (atmospheric pressure and at $60^{\circ} \mathrm{C}$ ) and the prevailing reaction pathway can easily be chosen only by controlling the amount of dissolved oxygen in the solution. Following the oxidation pathway, the phenol can be eliminated through advanced oxidation processes. On the other hand, following the hydrogenation pathway, phenol is transformed into cyclohexanol and cyclohexanone. These findings suggest that a single CMR can successfully be used in AOPs or hydrogenation reactions. The catalytic membrane reactors can successfully be used for $\mathrm{Cr}$ (VI) to $\mathrm{Cr}$ (III) reduction with only hydrogen as reducing agent. In all cases, the Catalytic Membrane Reactor with smaller pore size presented the best performance.Thus, it was demonstrated that the pore size is important as far as performance is concerned.

\section{ACKNOWLEDGMENTS}

V. Pinos wants to express her gratitude for the economic support given by the doctoral scholarship from SENESCYT, convocatoria abierta 2012, I fase, and the Universidad de Cuenca. 


\section{REFERENCES}

Andreozzi, R., Caprio, V., Insola, A. and Marotta, R., Advanced oxidation processes (AOP) for water purification and recovery, Catalysis Today, 53, Nro 1, 51-59 (1999). https://doi.org/10.1016/S09205861(99)00102-9

Apha, AWWA and Wef, Standard Methods for the Examination of Water and Wastewater. 22nd ed. Amer Water Works Assn. Washington DC (2012).

Caro, J., Catalytic Membrane Reactors - Lab Curiosity or Key Enabling Technology?, Chemie Ingenieur Technik, 86, Nro 11, 1901-1905 (2014). https:// doi.org/10.1002/cite.201400069

Cheng, L., Dai, Q., Li, H., and Wang, X., Highly Selective Hydrogenation of Phenol and Derivates over Pd catalysts supported on $\mathrm{SiO} 2$ and $\gamma-\mathrm{A} 12 \mathrm{O} 3$ in aqueous media. Catalysis Communications, 57, 23-28 (2014).

Claus, P., Berndt, H., Mohr, C., Radnik, J., Shin, E.J., \& Keane, M.A., Pd/MgO: Catalyst Characterization and Phenol Hydrogenation Activity, Journal of Catalysis, 192, Nro 1, 88-97 (2000). https://doi. org/10.1006/jcat.2000.2834

Dalmon, J.A., Cruz-López, A., Farrusseng, D., Guilhaume, N., Iojoiu, E., Jalibert, J.C., Miachon, S., Mirodatos, C., Pantazidis, A., RebeilleauDassonneville, M., Schuurman, Y. and Van Veen A., Oxidation in catalytic membrane reactors, Applied Catalysis A: General, 325, Nro2, 198-204 (2007).

Ghedini, E., Menegazzo, F., Signoretto, M., Manzoli, M., Pinna, F. and Strukul, G., Mesoporous silica as supports for $\mathrm{Pd}$-catalyzed $\mathrm{H} 2 \mathrm{O} 2$ direct synthesis: Effect of the textural properties of the support on the activity and selectivity, Journal of Catalysis, 273, 266-273 (2010).

Glória, R.M., Motta, T.M., Silva, P.V.O., Da Costa, P., Brandt, E.M.F., Souza, C.L., and Chernicharo, C.A.L., Stripping and dissipation techniques for the removal of dissolved gases from anaerobic effluents, Brazilian Journal of Chemical Engineering, 33, Nro 4, 713-721 (2016). https://doi.org/10.1590/01046632.20160334s20150291

Lin, K., Ceramic Membranes for Separation and Reaction. Wiley. England (2007).

Liu, H., Jiang, T., Han, B., Liang, S., and Zhou, Y., Selective Phenol Hydrogenation to Cyclohexanone Over a Dual Supported Pd-Lewis Acid Catalyst, Science, 326, Nro 5957, 1250-1252 (2009). https:// doi.org/10.1126/science. 1179713

Liu, J., Li, H., and Li, H., Liquid-Phase Selective Hydrogenation of Phenol to Cyclohexanone over $\mathrm{Pd}-\mathrm{Ce}-\mathrm{B} / \mathrm{Hydrotalcite}$ Catalyst, Chinese Journal of Catalysis, 28, Nro, 4, 312-316 (2007). https://doi. org/10.1016/S1872-2067(07)60029-1
Mahata, N., Raghavan, K.V., Vishwanathan, V., Park, C., and Keane, M.A., Phenol hydrogenation over palladium supported on magnesia: Relationship between catalyst structure and performance, Physical Chemistry Chemical Physics, 3, Nro 13，2712-2719 (2001). https://doi.org/10.1039/ B100237F

Maksimov, A.L., Kuklin, S.N., Kardasheva, Y.S., and Karakhanov, E.A., Hydrogenation of phenols in ionic liquids on rhodium nanoparticles, Petroleum Chemistry, 53, Nro 3, 157-163 (2013). https://doi. org/10.1134/S0965544113030043

Matos, J., and Corma, A., Selective phenol hydrogenation in aqueous phase on Pd-based catalysts supported on hybrid TiO2-carbon materials, Applied Catalysis A: General, 404, Nro 1-2, 103-112 (2011). https://doi.org/10.1016/j. apcata.2011.07.018

Miachon, S., and Dalmon, J.A., Catalysis in membrane reactors: what about the catalyst?, Topics in Catalysis, 29, Nro 1-2, 59-65 (2004).

Mohanty, S.S., Jena, H.M., Mohanty, S.S., and Jena, H.M. Biodegradation of phenol by free and immobilized cells of a novel Pseudomonas sp. NBM11, Brazilian Journal of Chemical Engineering, 34, Nro 1, 75-84 (2017). https://doi. org/10.1590/0104-6632.20170341s20150388

Morales, J., Hutcheson, R., Noradoun, C., and Cheng, I. F., Hydrogenation of Phenol by the $\mathrm{Pd} / \mathrm{Mg}$ and $\mathrm{Pd} / \mathrm{Fe}$ Bimetallic Systems under Mild Reaction Conditions, Industrial \& Engineering Chemistry Research, 41, Nro 13, 3071-3074 (2002). https:// doi.org/10.1021/ie0200510

Oller, I., Malato, S., and Sánchez-Pérez, J.A. Combination of Advanced Oxidation Processes and biological treatments for wastewater decontamination-A review, Science of The Total Environment, 409, Nro 20, 4141-4166 (2011). https://doi.org/10.1016/j.scitotenv.2010.08.061

Osegueda, O., Dafinov, A., Llorca, J., Medina, F., and Suerias, J., In situ generation of hydrogen peroxide in catalytic membrane reactors, Catalysis Today, 193, Nro 1, 128-136 (2012). https://doi. org/10.1016/j.cattod.2012.01.040

Osegueda, O., Dafinov, A., Llorca, J., Medina, F., and Sueiras, J., Heterogeneous catalytic oxidation of phenol by in situ generated hydrogen peroxide applying novel catalytic membrane reactors, Chemical Engineering Journal, 262, 344-355 (2015). https://doi.org/10.1016/j.cej.2014.09.064

Park, C., and Keane, M.A. Catalyst support effects: gas-phase hydrogenation of phenol over palladium, Journal of Colloid and Interface Science, 266, Nro 1, 183-194 (2003). https://doi.org/10.1016/S00219797(03)00171-1 
Pérez, Y., Fajardo, M., and Corma, A. Highly selective palladium supported catalyst for hydrogenation of phenol in aqueous phase, Catalysis Communications, 12, Nro 12, 1071-1074. (2011). https://doi.org/10.1016/j.catcom.2011.03.026

Pignatello, J.J., Oliveros, E., and MacKay, A., Advanced Oxidation Processes for Organic Contaminant Destruction Based on the Fenton Reaction and Related Chemistry, Critical Reviews in Environmental Science and Technology, 36, Nro 1, 1-84 (2006). https://doi. org/10.1080/10643380500326564

Pinos-Vélez, V.P, Dafinov, A., Medina, F., and Sueiras, J., Chromium(VI) reduction in aqueous medium by means of catalytic membrane reactors, Journal of Environmental Chemical Engineering, 4, nro 2, 1880-1889 (2016a). https://doi.org/10.1016/j. jece.2016.03.008

Pinos-Vélez, V.P., Crivoi, D.G., Medina, F., Sueiras, J.E., and Dafinov, A.I., New tuneable catalytic membrane reactor for various reactions in aqueous media, ChemistrySelect, 1, Nro 2, 1-3. (2016b). https://doi.org/10.1002/slct.201500005

Pinos-Vélez, V.P., Medina, F., and Dafinov, A., Preparation and Characterization of Catalytic Membrane Reactors, Revista de La Facultad de Ciencias Químicas, Nro 16, 1-14 (2017).

Pires, C.A., dos Santos, A.C.C., Jordão, E., Oxidation of phenol in aqueous solution with copper oxide catalysts supported on $\gamma-\mathrm{Al}_{2} \mathrm{O}_{3}$, pillared clay and $\mathrm{TiO}_{2}$ : Comparison of the performance and associated with each catalyst, Brazilian Journal of Chemical Engineering, 32, Nro 4, 837-848 (2015). https://doi.org/10.1590/01046632.20150324s00002232

Poyatos, J.M., Muñio, M.M., Almecija, M.C., Torres, J.C., Hontoria, E., and Osorio, F., Advanced Oxidation Processes for Wastewater Treatment: State of the Art., Water, Air, and Soil Pollution, 205, Nro 1-4, 187-204 (2010). https://doi.org/10.1007/s11270-009-0065-1

Remias, J., Pavlosky, T., and Sen, A., Catalytic hydroxylation of benzene and cyclohexane using in situ generated hydrogen peroxide: new mechanistic insights and comparison with hydrogen peroxide added directly., Journal of Molecular Catalysis A: Chemical, Nro 203, 179-192 (2003).

Rome, K., McIntyre, A., and Macclesfield, A., Intelligent use of Relative Response Factors in Gas Chromatography-Flame Ionisation Detection, Chromatography Today, 52-56 (2012).

Sánchez, M., and Tsotsis, T. Introduction. In Catalytic Membranes and Membrane Reactors. Wiley-VCH. (2002).
Shi, L., Goldbach, A., Zeng, G., and Hengyong, X., Direct $\mathrm{H}_{2} \mathrm{O}_{2}$ synthesis over $\mathrm{Pd}$ membranes at elevated temperatures, Journal of Membrane Science, Nro 348, 160-166 (2010).

Sikhwivhilu, L.M., Coville, N.J., Naresh, D., Chary, K.V.R., and Vishwanathan, V., Nanotubular titanate supported palladium catalysts: The influence of structure and morphology on phenol hydrogenation activity, Applied Catalysis A: General, 324, 52-61 (2007). https://doi.org/10.1016/j. apcata.2007.03.004

Terra, N.M., Lemos, C.O.T., da Silva, F.B., Cardoso, V.L., Reis, M.H.M., Characterisation of asymmetric alumina hollow fibres: Application for hydrogen permeation in composite membranes, Brazilian Journal of Chemical Engineering, 33, Nro 3, 567-576 (2016). https://doi.org/10.1590/01046632.20160333 s20150074

Tomoya, I., Tanaka, Y., Pacheco, D., Suzuki, T., Sato, K., Nishioka, M., Hamakawaa, S. and Mizukami, F., Direct production of hydrogen peroxide from oxygen and hydrogen applying membranepermeation mechanism, Chemical Engineering Science, Nro 65, 436-440 (2010).

Volkov, V., Petrova, I., Lebedeva, V., Roldughin, V., and Tereshchenko, G. Palladium-Loaded Polymeric Membranes for Hydrogenation in Catalytic Membrane Reactors. In Membranes for Membrane Reactors: Preparation, Optimization and Selection. Wiley. Singapour (2011).

Wang, Y., Yao, J., Li, H., Su, D., and Antonietti, M., Highly Selective Hydrogenation of Phenol and Derivatives over a Pd@Carbon Nitride Catalyst in Aqueous Media., Journal of the American Chemical Society, 133, Nro 8, 2362-2365 (2011). https://doi. org/10.1021/ja109856y

Watanabe, S., and Arunajatesan, V., Influence of Acid Modification on Selective Phenol Hydrogenation Over Pd/Activated Carbon Catalysts, Topics in Catalysis, 53, Nro 15-18, 1150-1152 (2010). https://doi.org/10.1007/s11244-010-9551-3

Westermann, T., and Melin, T., Flow-through catalytic membrane reactors-Principles and applications, Chemical Engineering and Processing: Process Intensification, 48, Nro 1, 17-28. (2009). https:// doi.org/10.1016/j.cep.2008.07.001

Zhu, J.F., Tao, G.H., Liu, H.Y., He, L., Sun, Q.H., and Liu, H.C., Aqueous-phase selective hydrogenation of phenol to cyclohexanone over soluble $\mathrm{Pd}$ nanoparticles, Green Chemistry, 16, Nro 5, 26642669 (2014). https://doi.org/10.1039/C3GC42408A 